\section{Contributo Econômico do Farmacêutico Clínico na Gestão e Auditoria em Serviços de Saúde: Relato de Experiência.}

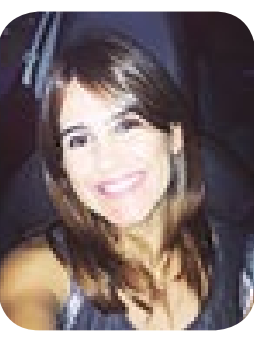

\section{RESUMO}

O trabalho aborda a importância da atuação do farmacêutico clínico sob um ponto de vista econômico, através de um relato de experiência de um Serviço de Farmácia Clínica em um hospital de grande porte em Salvador-BA. A relevância do tema e a escassez de estudos que façam uma interrelação entre o farmacêutico e a economia gerada a partir de suas intervenções no sistema de saúde hospitalar foram os motivos que levaram a essa construção. Tem como objetivo contribuir para o conhecimento dos ganhos em saúde decorrentes da prática do farmacêutico clínico, descrever as ações e resultados obtidos para garantir a segurança do paciente, reduzir custos e dar suporte à equipe multiprofissional e a auditoria interna, contribuindo de forma estratégica na gestão farmacoterapêutica, clínica/assistencial e econômica da instituição. Comprovadamente há uma redução de custos agregada às intervenções realizadas, mas a contribuição deste profissional no que se refere à assistência e segurança do paciente é imensurável. $O$ trabalho provocou a reflexão sobre o papel do farmacêutico clínico e seus impactos do ponto de vista clínico e econômico.

PALAVRAS-CHAVE: gestão em saúde. Farmácia clínica. Custos em saúde. Glosas.

\section{INTRODUÇÃo}

A redução de custos tornou-se uma das maiores preocupaçôes dos gestores das instituiçoes de saúde de todo o País, tanto na administração pública quanto no setor privado. No Sistema Público de Saúde (SUS) a discussão atual gira em torno do financiamento da assistencia e principalmente sobre o congelamento por vinte anos, em investimentos nas áreas de saúde e educação, com reajuste corrigido pela inflação do ano anterior. Nos últimos anos, aumentaram-se gastos presentes e futuros, em diversas políticas públicas, sem levar em conta as restrições naturais impostas
Bárbara Kelly Oliveira da Silva ${ }^{1}$

pela capacidade de crescimento da economia, ou seja, pelo crescimento da receita (PEC 241/2016). No setor privado, as instituições tiveram que aprender a associar baixos custos com excelência de qualidade para os seus clientes; gastar menos e melhor deve ser um dos objetivos a ser seguido. Consequentemente, as instituições de saúde têm sido compelidas a se organizarem como empresa, desenvolvendo visão de negócio para sobreviverem às mudanças no mercado. Esta tendência mundial exigiu dos profissionais envolvidos habilidade na análise de custos para a prestação de serviços de saúde e a utilização constante da auditoria de contas hospitalares como ferramenta de controle $e$ dução de gastos (ROSA, 2013).

No âmbito das organizações hospitalares, é importante observar todos os processos assistenciais que tem impacto direto no faturamento das contas; dentificar fragilidades na prestação do cuidado que represente desperdicios e expõe o paciente ao risco, bem como a analise do uso racional de materiais medicamentos, é fator primordial no contexto da gestão hospitalar.

Estudos realizados pela Universidade Federal de Minas Gerais apontam que erros em hospitais podem matar mais gente no Brasil do que câncer; segundo estimativa, falhas durante internações podem ter matado até 434 mil pessoas no Brasil no ano passado. Prejuízo com erros podem chegar a $\mathrm{R} \$ 15$ bilhões (IESS, 2016). Outra pesquisa do Instituto de Estudo de Saúde Suplementar, realizada por uma autogestora de plano de saúde, relata que os gastos com materiais e medicamentos são o principal fator para o aumento dos custos de planos. Enquanto os valores destinados aos medicamentos subiram $59,9 \%$ no periodo analisado, os gasto com materiais avançaram 120,4\%; isso é explicado, em parte, pela introdução de novas tecnologias em saúde, mais caras do que as anteriores, muitas vezes utilizadas de forma indiscriminada e sem medições de eficácia, segurança ou qualidade (SANTOS, 2013).
O consumo de saúde é composto por basicamente três estruturas diferentes: medicamentos; honorários (médicos, dentistas e auxiliares dos serviços médicos) e gastos em hospitalização e tratamento, estes encabeçam as despesas do consumo em saúde (ZUCCHI, 2000). No ambiente hospitalar, a terapia medicamentosa é utilizada para tratamento das doenças e manutenção da saúde. No entanto, os pacientes hospitalizados e que fazem uso de múltiplos medicamentos encontram-se mais vulneráveis à ocorrência de eventos adversos (OLIVEIRA, 2014). Atualmente, a prevalência de hospitalizações relacionadas a medicamentos é de 4,3\%, sendo $59 \%$ delas evitáveis. A incidência de internação por Eventos Adversos a Medicamentos (EAM) evitáveis é estimada em 4,5 por 1.000 pessoas/mês. Outros dados apontam que $28 \%$ de todas as consultas de pronto-atendimento são relacionadas a medicamentos, sendo $70 \%$ delas evitáveis e $24 \%$ delas são futuras causas de internações hospitalares (CORRER, 2011).

Nos últimos anos, o gasto farmacêutico vem tornando-se uma ameaça à sustentabilidade dos sistemas de saúde de muitos países. O orçamento crescente destinado à provisão dos medicamentos tem competido com outras grandes prioridades no setor (OMS, 2002). Ademais, os medicamentos são causas de internamentos ou eventos que provocam danos ao paciente ou prolongam a permanência destes no hospital; nestes casos, o farmacêutico pode contribuir significativamente para a redução destes custos. Entendendo-o como o profissional de referência quando se trata de medicamentos, já que $\mathrm{o}$ assunto é pertinente a seu campo de atuação, ele também é o responsáve pelo Ciclo da Assistência Farmacêutica, processo que compreende a seleção, aquisição, armazenamento, distribuição, dispensaçăo de medicamentos, a prescrição, a orientação e o acompanhamento do uso; sendo assim, o farmaceutico, inserido nestas etapas e junto à equipe multidisciplinar nas areas pode reduzir os gastos desnecessários, bem como diminuir a exposição de pacientes aos riscos inerentes aos produtos e processos assistenciais, sugerindo mudanças.

Além disso, o aumento dos gastos referentes a materiais e medicamentos pode ser reflexo de vários fatores, como a expansão de cobertura, surgimento de novos fármacos, dinamismo epidemiológico, envelhecimento da população, expectativas dos pacientes e o uso inadequado de fármacos em diversas situaçoes clínicas. Salienta-se ainda que esses gastos disponibilizam uma gama variável e imensa de medicamentos para o sistema de saúde, o que potencializa a ocor- rência de irracionalidade na sua utilização por parte indivíduos e profissionais de saúde (MOTA, 2008) Frente ao atual cenário da saúde, ressalta-se a importância de adotar uma abordagem econômica para as questões ligadas ao uso racional de medicamentos. A relevância do tema e a escassez de estudos que façam uma interrelação entre o farmacêutico e economia gerada a partir de suas intervenções e contribuições no sistema de saúde hospitalar tornam necessárias as stantes descrições sobre o assunto.

Assim, este trabalho tem como objetivo relatar a ex periência de um Serviço de Farmácia Clínica (SFC) e as atividades realizadas para garantir a segurança do paciente, reduzir custos e dar suporte à equipe multiprofissional e auditoria interna, contribuindo de forma estratégica na gestão farmacoterapêutica, clínica/assistencial e econômica da instituição.

O resultado deste estudo pode sensibilizar os gestores das instituições no sentido de perceberem quão benéfica e a integração do farmacêutico clínico junto às equipes de saúde e seus impactos.

\section{MATERIAIS E MÉTODOS}

Trabalho retrospectivo e descritivo que visa identificar os resultados alcançados desde a implantação do Serviço de Farmácia Clínica, através de um relato de experiência, no qual se busca demonstrar as rotinas e atividades dos farmacêuticos que contribuíram na gestão, redução de custos e suporte às equipes multiproissional e de auditoria interna.

Para a realização deste trabalho utilizou-se também, como embasamento téorico, artigos datados de 2002 a 2016, das bases de dados do Scientific Eletronic Library Online (SciELO), Lilacs, obras de língua portuguesa e espanhola, leis e portarias vigentes no Pais, alem de reportagens e publicaçôes atualizadas que traziam as palavras-chave: auditoria em saúde; gestáo em saúde, auditoria farmacêutica; custos em saude, glosas; ou abordavam o titulo proposto: Pape do Farmacêutico em Gestão e/ou Auditoria. Foram excluídos os artigos que apresentavam informações repetidas ou disponíveis em outros artigos.

\section{Caracterização do local do estudo}

Com o intuito inicial de atender às exigências do mercado e de contribuir para a prevenca de morbimortalidade por meio da identificação, resolução e prevenção de Problemas Relacionados a Medicamentos (PRM), foi implantado em 2014 pela diretoria e gerência medica do Hospital o Serviço de Farmácia Clínica (SFC). 
trabalho foi realizado em um hospital de grande porte em Salvador-BA, certificado pela ONA com nivel 3 de excelência; filantrópico e de ensino, com 549 leitos, sete unidades de terapia intensiva, sendo cinco adultos e duas pediatricas, que totalizam 85 leitos, em 39 especialidades, em serviços médicos de alta complexidade e apoio diagnostico e terapêutico, exceto obstetricia. Presta atendimento principalmente nas áreas de Cardiologia e Ortopedia (reconhecidos como centro de alta complexidade pelo Ministério da Saúde), Oncologia, Pediatria e Neurologia, atuando com excelencia tambem em diversas outras especiaidades. Realiza 12 mil cirurgias e 145 mil consultas e procedimentos ambulatoriais ao ano e dispõe ainda de 4 unidades de Pronto Atendimento: adulto, infantil, ortopedia e otorrinolaringologia. A assistência ambulatorial e hospitalar contempla os pacientes particulares e conveniados às principais operadoras e planos de saúde, alem dos usuários encaminhados para atendimento pelo Sistema Unico de Saúde (SUS). O hospital conta com equipe multidisciplinar como: médicos, farmaceulicos, fisioterapeutas, nuthicionistas, enfermeiros, fonoaudiólogos, assistentes sociais e psicólogos, atuando de forma integrada.

Os serviços de farmácia dispõem de uma equipe de 22 farmacêuticos, sendo: 4 coordenadores distribuídos nas diferentes áreas: Farmácia de Logística e Suprimentos, Serviço de Farmácia Clínica, OPME (Órteses, Próteses e Materiais Especiais) e Oncologia, sendo 7 farmacêuticos na Gestão de Logística e Suprimentos. Destes, dois em dias alternados no serviço noturno; 4 no Serviço de Oncologia, 2 no Serviço de Nutrição Parenteral; 1 na Central de Abastecimento Farmacêutico; 1 no Ensino e Pesquisa e 3 no Serviço de Farmácia Clínica, este último, objeto desse relato, conta também com 6 estagiários de nível superior do curso de farmácia.

mplantação do Serviço

A estrutura física do Serviço de Farmácia Clínica (SFC) e composta por uma sala com computadores, livros e acesso a sites de busca, como o UpToDate, Drugs.Com, Micromedex. As evoluções dos pacientes e as prescrições médicas são elaboradas em sistema informatizado.

Em 2014 ocorreu a implantação e divulgação do serviço ao corpo clínico da instituiçáo. O desafio imediato seria o processo de acreditação pelo qual passava todo o hospital. Neste primeiro momento, SFC desenvolveu alguns materiais de orientações à equipes e realizou treinamentos in locu has unidades assistenciais sobre a utilização de medicamentos via sonda e campanha de incentivo as notificaçôes de reaçoes adversas; alem disso, foram definidas atividades em setores específicos. No pronto atendimento adulto foi realizada a conciliaçáo medicamentosa' de todos os pacientes com idade igual ou superior a 60 anos internados em leitos virtuais, acompanhamento dos pacientes pediatricos por criticidade, O parse e a farmacovigilâncią.

O passo seguinte envolveu a participação do serviço na parametrização de informações relacionadas a medicamentos no novo sistema informatizado. Foram cadastrados um total de um mil e quatrocentos medicamentos padronizados do hospital, bem como todos os insumos necessários à sua administração que eram dispensados via sistema, considerando dose prescrita, forma de aplicação, reconstituição e diluição do medicamentos injetáveis de acordo com o manual de dilucição institucional. Orientações importantes sobre os medicamentos, como a forma de administração, flebite, medicamentos potencialmente perigosos, interações medicamentosas e fármaco-nutrientes, foram dastradas no sistema.

Para atender exclusivamente a prescrição pediátrica e com a finalidade de reduzir o número de erros $\mathrm{d}$ medicação relacionados à prescrição, administração e dispensação de medicamentos foi desenvolvido pela Tecnologia da Informação do hospital, com suporte do MV e apoio do SFC, o Sistema Auxiliar de Cadastro (SAC), integrado ao sistema MV. No SAC, os cálculos de dose por quilo de peso do paciente, de reconstitução e diluição, conforme padrão de diluição pediátrico institucional, são automatizados, os insumos e medicamentos são dispensados de acordo com os resultados destes cálculos. Além disso, o tempo de administração padronizado na pediatria foi inserido no sistema, a fim de garantir maior segurança na infusão dos medicamentos, bem como uniformizar as rotinas. Não houve custo adicionais para a implantação do sistema auxiliar.

Devido à necessidade de se adaptar à realidade da instituição, foi necessário um novo delineamento das atividades, buscando atender unidades que fossem mais criticas, principalmente pelo número limitado de farmacêuticos. Os critérios de elegibilidade passaram

'Conciliação medicamentosa: consiste na obtenção de uma lista completa, precisa, atualizada dos medicamentos que cada paciente utiliza em casa, que deve ser comparada com as prescrições médicas feitas na admissão, transferência, consultas ambulatoriais e alta hospitalar, visando assegurar a terapêutica do tratamento individualizado (CFF, 2010), observando o quadro clínico atua l.

${ }^{2}$ Farmacovigilância: e a ciència e atividades relativas à identificaçáo,
quer problemas relacionados ao uso de medicamentos. (ANVISA, 2009)

por mudancas ao longo desses anos e foram definidos onsiderando a criticidade das unidades e dos pacienpor 1. Unidades pedítrics, que contam com uma cêutica e um estagiário.

2. Unidades de terapia intensiva adulto, que são divididas entre duas farmacêuticas, priorizando pacientes das linhas do cuidado (Síndrome Coronariana Aguda e Acidente Vascular Cerebral), idosos, pacienS SUS e nefropatas.

3. Unidades abertas do SUS, onde as atividades são desenvolvidas pelos estagiarios sob supervisão Asmaceuticas referência adultos.

As farmacêuticas realizam o Seguimento Farmacoterapêutico $^{3}$ (SF) e orientações à equipe, além de responder às demandas espontaneas; os estagiários fazem anallise de prescriçôes nas unidades SUS, rotinas gerenciadas, conciliação medicamentosa e farmacovigilancia, objetivando maior segurança, uso racional dos medicamentos e otimização da terapia sem prejuízos ao paciente.

Importante ressaltar que, ao longo desses 2 anos houve também uma preocupação constante da equipe com a produção de materiais informativos para as áreas assistenciais, treinamentos e reorientações de trabalho, revisão dos manuais já existentes e criação de novos.

Os indicadores do Serviço seguem as referências do Proqualis e de grandes hospitais nacionais e mundiais, de acordo com as atividades realizadas:

1. Total de paciente perfil $x$ acompanhado $x$ meta

2. Intervenções realizadas $x$ acatadas

3. Intervenções de antimicrobianos

4. Taxa de conciliação medicamentosa

5. Farmacoeconomia

6 . Atendimentos a dúvidas

7. Farmacovigilância

\section{RESULTADOS E DISCUSSÃO}

Muitos estudos publicam dados quantitativos refeentes as prescriçoes analisadas, problemas relacionados a medicamentos encontrados e intervenções realizadas, que indiretamente já demonstram o quanto o farmacêutico pode contribuir na assistência ao paciente e à equipe. Contudo, a proposta deste relato é mostrar o quanto essas açoes representam de economia para a instituição.
Os dados são manuais, o sistema ainda não conemplou os indicadores do SFC, contudo, neste último semestre, houve uma sensibilização da equipe, farmacêuticos e estagiários, para a importância de registrar as ações e transformá-las em valores; assim, houve uma melhor adesão e conscientização de todos para me registros. Abaixo, gráfico de intervenções pontuais realizadas.

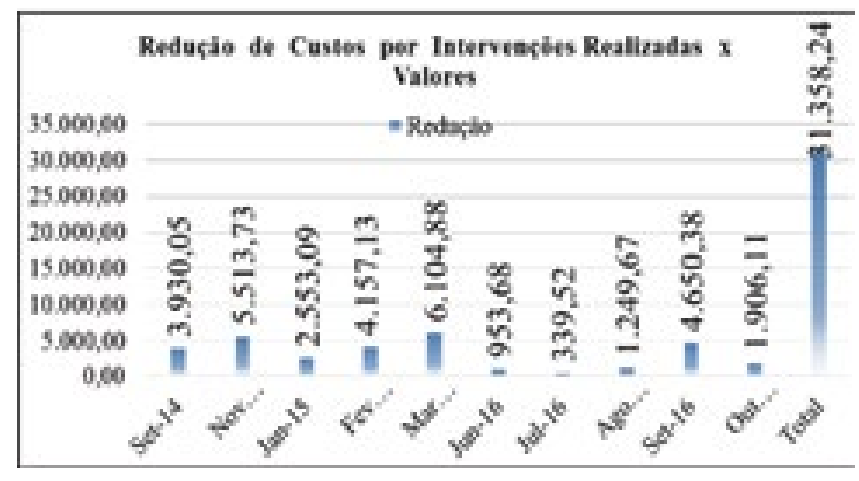

Gráfico 1 - Redução por Intervenção em Valores

As intervenções normalmente incluem redução po ajuste de dose, por mudança da posologia, mudança do esquema terapeutico, mudança da forma tarmacêutica (venoso para oral, ou outras apresentações) e ate suspensão do medicamento, dentre outras, conforme a seguir:

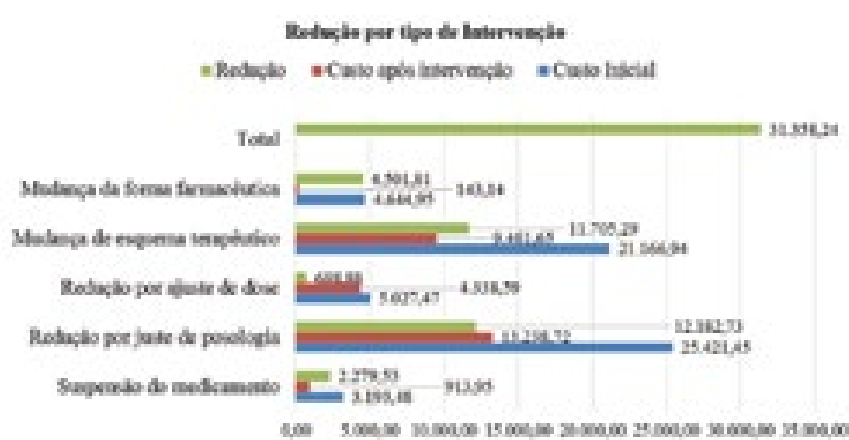

Gráfico 2 - Tipo de intervenção

${ }^{3}$ Seguimento farmacoterapêutico individualizado é a prática profissional na qual o farmacêutico se responsabiliza pelas necessidades do paciente relacionadas com os medicamentos, mediante a detecção, prevenção e resolução de Problemas Relacionados a Medicamentos (PRM), de forma continuada, sistematizada e documentada, em colaboração com o próprio paciente e com os demais profissionais do siste-
ma de saúde, a fim de alcançar resultados concretos que melhorem a qualidade de vida do paciente. (ANGONESI, D; SEVALHO, G., 2010) 
Houve uma redução de 31,358,24 (trinta e um mil, trezentos e cinquenta e oito reais e vinte e quatro centavos) em intervenções pontuais realizadas, guardando-se a existência de dados subnotificados; assim, se pensarmos em um farmacêutico com dedicação exclusiva para a clinica em unidades criticas para segurança e consumo, certamente teríamos resultados mais dedignos e significativos.

Para mensurar a redução de custos é utilizada a planilha abaixo, com dados e dias de permanência do paciente após intervenções com valores dos medicamentos:

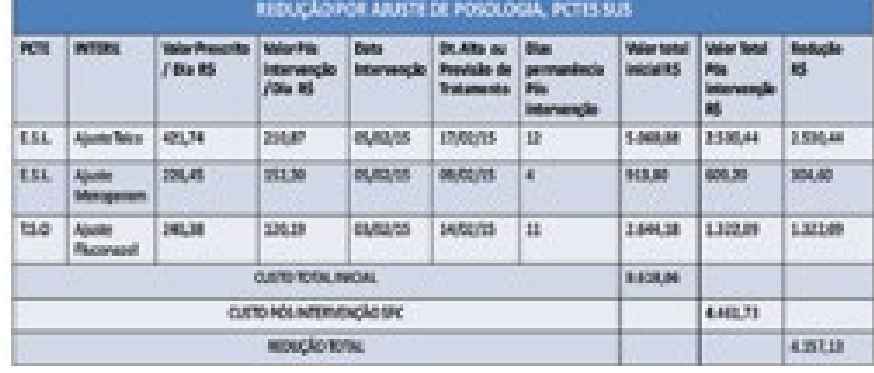

Figura 1 - Planilha Controle de Redução de Custos por Intervenção Realizada - SUS, SFC - Fev/15.

Todos as intervenções são discutidas com equipe médica e de enfermagem e são realizadas considerando quadro clínico do paciente.

Por ser hospital filantrópico e de ensino, presta atendimento a pacientes do Sistema Unico de Saúde provenientes das UPAs (Unidades de Pronto Atendimento) via Central de Regulação do Estado ou através de postos de saúde, também via Regulação Municipal, para fazer procedimentos cirúrgicos ambulatoriais. Contudo, chegam com comorbidades diversas, que prolongam seu internamento no hospital. Muitas vezes, por não recorrerem às redes básicas de saúde para controle de sua doença, no momento do internamento, faz-se necessário realizar mais exames ou fazer uso de medicamentos para prepará-lo ou estabilizá-lo para o procedimento cirurgico, o que onera o atendimento. A contribuição do SFC para a instituição frente aos convênios são muito subjetivas, não se mensura intervenções que implicam em indicadores qualitativos, ou seja, no melhor resultado clínico do paciente, giro de leitos, ou melhor resposta terapêutica. Contudo, no Seguimento Farmacoterapêutico, o olhar diferenciado deste profissional em relação ao uso dos medicamentos agrega segurança ao cuidado assistencial.

No Seguimento Farmacoterapêutico (SF) é realizada a avaliação da prescrição médica, observando problemas relacionados a medicamentos, o uso do antibióticos/stewardship (indicação, cultura, dose, tem po de tratamento e descalonamento da terapêutica) com a função rena do paciente, avalia a presença de interações medicamentosas, verifica alergias, reaçoes adversas, duplicidade de medicamentos que geram glosas, posologia indicação, faz a concillação medicamentosa, monitorização clínica e laboratorial e a alta orientada aos pacientes da Sindrome Coronariana Aguda (SCA).

No SF, o farmacêutico clínico consegue identificar reações adversas ou interações medicamentosas graves ao uso de medicamentos que normalmente não são associadas pela equipe médica; esse é o diferencial deste profissional no cuidado ao paciente. Reações como: disfunção renal (mais comum), convulsões, parada cardiorrespiratória (PCR), distúrbios visuais, flebite química grave, Necrólise Epidérmica Tóxica (NET) e ate obito são causadas por medicamentos e nem sempre vistos pela equipe como causa da piora clínica do paciente; assim inicia-se um processo de investigação, com exames e medicamentos de alto custo, que onera o sistema de saúde.

O sistema de prescrições pediátricas, o SAC - Sistema Auxiliar de Cadastros, também contribui para a redução de glosas e traz segurança no processo. A mudança do sistema informatizado vigente no hospital suscitou não só a possibilidade de melhoria da prescrição médica, como também de todos os cálculos para a administração dos medicamentos, os quais demonstravam serem processos críticos na rotina das unidades pediátricas da instituição (SANTANA, 2015). Abaixo exemplo da prescrição na tela de aprazamento da enfermagem e cálculos:

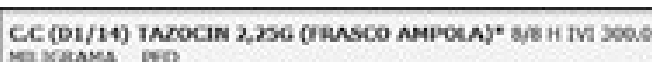

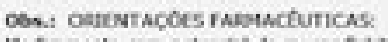

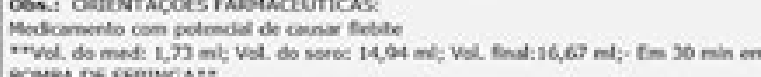

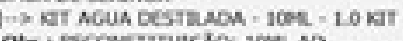

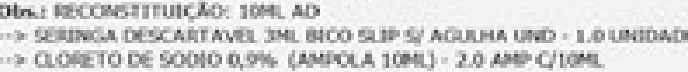

Figura 2 - Tela de aprazamento de medicamentos da enfermagem com os cálculos e informações sobre medicamentos.

Essa padronização uniformizou as ações de todas as unidades e embasou as cobranças dos insumos dos medicamentos junto aos convênios, através de ustificativas tecnicas, reuniôes e discussões com os auditores de grandes operadoras de planos de saúde.
Após implantação do SAC, não foram observados rros relacionados aos cálculos de reconstituição e diluição de medicamentos. Erros nos cálculos de dose foram observados imediatamente após a implantação do sistema, devido à adaptação dos prescritores quanto ao novo padrão adotado para a prescrição pediátrica. Após 5 meses de implantação, percebem-se erros pontuais durante avaliação da prescrição pela farmácia clínica, os quais são discutidos e corrigidos caso a caso. Em relação aos erros de dispensação, ainda não foi possivel identificar se houve redução, devido aos ajustes e desenvolvimentos que estão sendo realizados nas rotinas de dispensação da farmacia em âmbito institucional. No entanto, espera-se uma redução de tais erros, com consequente redução do volume de devolução de medicamentos e materiais. Desta forma - SAC tem demonstrado ser uma ferramenta inovadora, que proporciona maior segurança na prescrição e administração de medicamentos nos pacientes pediátricos (SANTANA, 2015).

$\mathrm{Na}$ pediatria, as unidades abertas totalizam uma média de 40 pacientes/dia. Nestas unidades, as prescrições são analisadas diariamente para verificar possíveis Problemas Relacionados a Medicamentos (PRM), como: via ou frequência de administração inadequada; dose inexistente e/ou acima ou abaixo da dosagem usualmente prescrita para o peso do paciente pediátrico; prescrição de medicamentos com mesma ação farmacológica ou outras não conformidades que necessitem de esclarecimento junto à equipe médica. Abaixo, os dados da analise técnica das prescrições pediátricas no periodo de maio a outubro de 2016 e intervenços realizadas pontualmente em oulubro/46:

Gráficos Unidades Abertas Pediátricas, SFC/2016

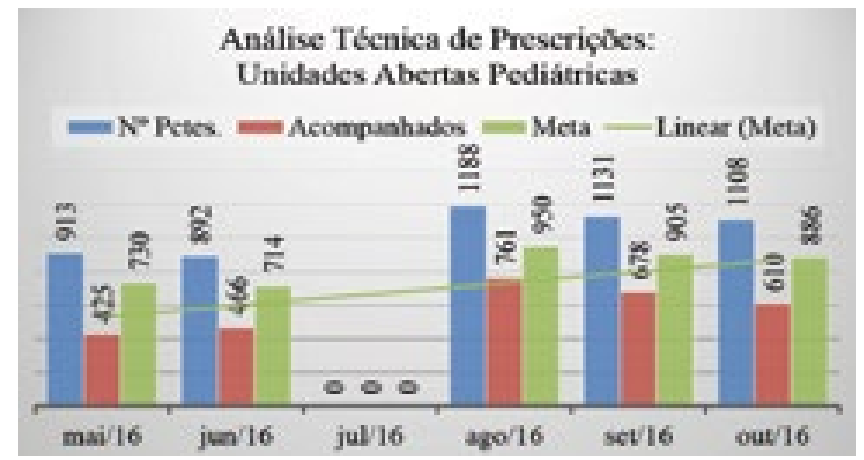

Gráfico 3 - Análise técnica de prescrições
Intervenções realizadas:

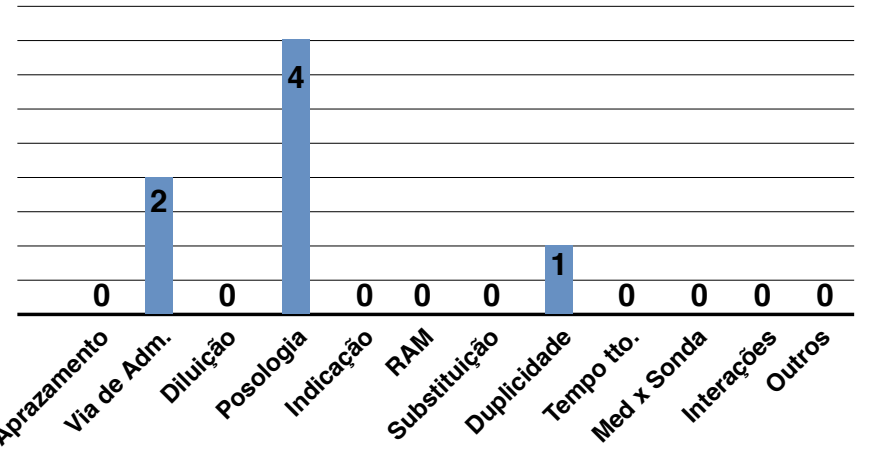

Gráfico 4 - Intervenções realizadas

Resultado das Intervenções: Unidade

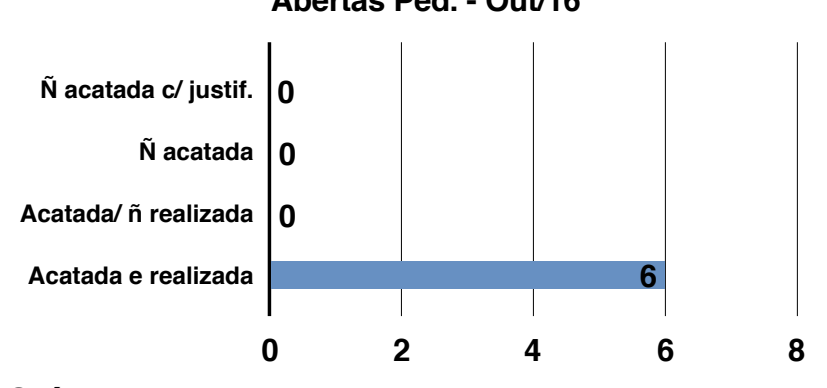

Gráfico 5 - Resultado das intervenções

Como já esclarecido anteriormente, houve uma subnotificação de muitos dados e na pediatria em específico, houve muitas intervençoes registradas como evolução no sistema que não foram inseridas nas planilhas, principalmente após a mudança do sistema que ocorreu em fevereiro/16. Em julho não houve acompanhamento, em função do recesso de estágio.

O SFC em números, em 2 anos e oito meses de trabalho:

1.400 medicamentos cadastrados e respectivos insumos necessários a administração; informaçōes sobre os medicamentos: se alto risco, flebite, sonda, interações, dentre outros dados importantes.

11 pareceres técnicos para a auditoria interna embasar cobranças aos convênios ou recursar glosas.

06 reuniões com auditores externos de grandes convênios para apresentação e discussão do Manual de Diluição da Pediatria.

07 informes técnicos às equipes sobre o uso de medicamentos. 
16 publicações de materiais informativos - 15 treinamentos às equipes assistenciais em sala ao ano.

- 108 introdutorios sobre os serviços de farmácia. Todas essas ações diminuem as possibilidades de glosas e contribuem direta ou indiretamente no resultado financeiro do hospital. Minimizar os custos dos recursos utilizados da farmacoterapia, sem comprometimento dos padrões de qualidade, e também um Cos objetivos para o uso racional dos medicamentos.

Comparando as atividades clínicas deste relato às de publicaçoes em artigos diversos e aos grandes hospitais, como o Einstein, por exemplo, elas não se diferem muito no perfil e rotinas e todas têm um único objetivo: a segurança do paciente.

Einstein, dentre as atividades desenvolvidas, estão: 1. Visita clínica multiprofissional ao leito $e$ poio à terapêutica nas questões pertinentes.

2. Visita específica do profissional farmacê

tico ao paciente em casos predeterminados.

3. Análise da prescrição médica onde o far-

macêutico verifica a descrição de medicamen-

to, dose do medicamento e ajustes específicos (idosos, homeopatas, nefropatas), via de administração (oral, sonda nasoenteral, intravenosa, intramuscular), frequência, diluição, volume, tempo de infusão, estabilidade, compatibilidade, interações medicamentosas, alergias, medicamentos associados e risco de queda.

4. Substituição de medicamentos não padro-

izados.

5. Atendimento a dúvidas e questionamentos

à equipe multiprofissional.

6. Farmacovigilância

7. Participação em comissões internas do

hospital.

8. Participação em protocolos clínicos.

(FERRACINI, 2011)

Impacto Clínico e Econômico do Acompanhamento Farmacoterapêutico

Conforme abordado na introdução, a morbimortalidade relacionada a falhas na farmacoterapia assume dimensões consideráveis merecedoras, sem dúvida de intervenção e minimização, também pelo farmacêutico.

A presença do farmacêutico clínico nas instituições de saúde e ambulatórios, realizando o acompanhamento farmacoterapêutico, através da identificação, resolução e prevenção de resultados clínicos negativos da farmacoterapia, seja por inefetividade ou inse- gurança, constitui uma prática profissional cujo contributo para a redução dos custos associados a esta morbimortalidade é significativo.

São muitos os estudos que demonstram o impacto clínico e econômico da intervenção do farmacêutico, sobretudo no âmbito internacional.

O "Minnesota Pharmaceutical Care Project" foi, provavelmente, um dos primeiros estudos que demonstraram os resultados clínicos e econômicos da intervenção farmacêutica; teve início em 1992 e três anos mais tarde, analisando os resultados clínicos obtidos entre 1994 e 1995, foi registado um aumento de 15\% no número de doentes que alcançaram o seu objetivo terapêulico (de $55,5 \%$ para $70,4 \%$, dos doentes envolvidos). No que se refere à economia gerada, esta foi estimada, para os doentes com idade igual ou superior a 65 anos, numa proporção de 11:1, isto é, por cada dólar investido na prática de Pharmaceutical Care, o Potencial, de $11 \mathrm{~d}$ ares (CONDINHO, 2014)

Além destes, muitos outros estudos relacionados ao acompanhamento de pacientes hipertensos, diabe ticos, com problemas cardíacos e polimedicados demonstraram claro benefício clínico através do controle da doença e adesão à farmacoterapia.

\section{CONSIDERAÇÕES FINAIS}

O Serviço de Farmácia Clínica desenvolve açõe que implicam na segurança e melhoria da terapia farmacológica do paciente. Há muitos problemas que acometem os pacientes e que podem estar diretamene relacionados ao uso de medicamentos, a exemplo de reações adversas graves, subdose ou sobredose de medicamentos, erros na prescrição, indicação de antibióticos, administração ou infusão de medicamentos, interações medicamentosas moderadas a graves, que prolongam o tempo de internamento e oneram os gastos com a saúde.

E indiscutível a mais-valia clínica e econômica da inserção do farmacêutico clínico na prática de cuidados relacionados ao uso de medicamentos, seja em ambiente hospitalar ou fora dele. Em nível ambulatorial, a presença do farmaceutico, através de seus consultórios, realizando o acompanhamento farmacoterapêutico, ja e uma realidade no mercado de trabalho. No ambiente hospitalar e imprescindivel o reconhecimento deste profissional, sobretudo oferecendo condições adequadas para que o farmacêutico clinico exerça suas atividades em sua plenitude e de forma continuada. Reconhece-se, contudo, que mais estudos serão necessários, principalmente em nível nacional, para que se possa retirar conclusões mais consistentes e adequadas à realidade hospitalar.

O tras a realidade hospitalar. estores de repensar o papel do farmacêutico e seus impactos do ponto de vista clínico e econômico, com o intuito de promover recursos humanos compatíveis com a estrutura organizacional, para que mais se pos aze em beneficio do paciente e da instituição.

Sugere-se a realização de outros estudos que contemplem açoes e resultados provenientes do oferecimento de SFC em instituiçoes hospitalares do Brasil, com demonstração de resultados financeiros decorrentes de suas intervenções.

\section{REFERÊNCIAS}

ANAHP. Observatório ANAHP. 2016. Disponível em: < http://anahp.com.br/produtos-anahp/observatoMolobservatorio-2016> Acesso em. 29 out. 2016.

ANVISA. O que e farmacovigilância? 2009. Disponível em: http://portal.anvisa.gov.br/o-que-e-farmacovigilancia- , acesso em 26/11/2016.

ANGONESI, D; SEVALHO, G. Atenção Farmacêutica: fundamentação conceitual e crítica para um modelo brasileiro. Revista Ciência e Saúde Coletiva $\mathrm{n}$. 15, 2010. Disponivel em: http://www.scielo.br/p 15s3/v15s3a35.pdf , acesso em 26/11/16

BISSON, M. P. Farmácia Clínica e Atenção Farmacêutica. 2.ed. Barueri: Manoli, 2007. 365 p.

BORGES, M. R. et al. A gestão por processos para a melhoria da qualidade dos serviços: $O$ caso de um hospital. 2012

CONDINHO, M.S.L. Estudo do impacto econômico do acompanhamento farmacoterapêutico. Julho/2014 Tese de Doutoramento em Farmacia na especialidade de Farmacologia e Farmacoterapia. Universidade de Coimbra.

CORRER, C.J. et al. Assistência farmacêutica integrada ao processo de culdado em saúde. gestáo clínica do medicamento. 2011. Disponivel em: http://scielo. iec.pa.gov.br/pdf/rpas/v2n3/v2n3a06.pdf, acesso em 24 de novembro de 2016

CFF. Conciliação medicamentosa. Farmácia Hospitalar, Pharmacia Brasileira, Conselho Federal de Farmácia, 2010. Disponível em: http://www.cff.org.br/, acesso em 24/11/2016.

CFF. Farmacovigilância. Farmácia Hospitalar, Pharmacia Brasileira, Conselho Federal de Farmácia, 2010. Disponível em: http://www.cff.org.br/, acesso em 26/11/2016.

FERRACINI, F. T. et al. Farmácia clínica: Segurança na prática hospitalar. São Paulo: Ed. Atheneu, 2011, p 24.
FORBES BRASIL Com gastos de R\$ 450 bi, saúde representa $10 \%$ do PIB do Brasil. Disponível em: http://anahp.com.br/noticias/noticias-do-mercado/ com-gastos-de-r\$-450-bi-saude-representa-do-brasil, Fonte: Revista Forbes Brasil Acesso em 08/11/16.

IESS. Falhas consomem mais de $\mathrm{R} \$ 15$ bi da saúde privada por ano no Brasil; disponível em http://www. iess.org.br/?p=blog\&id=273, acesso em 17/11/2016,

MOTA, D.M. et al. Uso racional de medicamentos: uma abordagem econômica para tomada de decisões Cencia e Saúde Coletiva 13: 589-601,2008.

OLIVEIRA, A.D. et al. Glosas de materiais e medicamentos em um hospital privado na cidade de BrasiDistrito Federal. 2012.

OLIVEIRA, M.R. et al. Estratégias para promover segurança do paciente: da identificação dos riscos às práticas baseadas em evidências. Escola Anna Nery Revista de Enfermagem 18, Jan-Mar 2014, disponi vel em:http://www.scielo.br/pdf/ean/v18n1/141

OLIVEIRA, S.A.R; JUNGES, F. Papel do profissional farmaceutico no ambito da assistência farmacêutica. Disponivel em: http://www.cpgls. pucgoias.edu.br/8mostra/Artigos/SAUDE\%20 \%20BIOLOGICAS/PAPEL\%20DO\%20PROFISSIONAL\%20FARMAC\%C3\%8AUTICO\%20 N O \% $20 \%$ C $3 \% 82 \mathrm{MB}$ I T O \% $20 \mathrm{DA} \% 20$ ASSIST\%C3\%8ANCIA\%2OFARMAC\%C3\%8AUTICA. pdf , acesso em: 20/11/16.

OMS. Promoción del uso racional de medicamenos: componentes centrales. Perspectivas políticas sobre medicamentos de la OMS. Set/2002. Disponvel em: http://apps.who.int/medicinedocs/pdf/s4874s/ s4874s.pdf , acesso em 26/11/2016.

PEC 241/2016, Altera o Ato das Disposições Conslitucionais Transitorias, para Instituir o Novo Regime Fiscal. Disponivel em http://www.camara.gov. $\mathrm{br} /$ proposicoesWeb/fichadetramitacao?idProposic $\mathrm{ao}=2088351$. Acesso em 12/10/2016 as 20:35.

Revista Exame.com (on-line): Erros em hospitais podem matar mais gente no Brasil do que câncer. <http://exame.abril.com.br/brasil/erros-em-hospitais-podem-matar-mais-gente-no-brasil-do-que-cancer/> esso em 29/10/2016, às 18:14.

ROSA, C.D.P, et al. Auditoria de contas hospitalares: Análise dos principais motivos de glosas em uma instituição privada. 2013.

SANTANA, E. F. Criação e implantação de um sistema para automatização de cálculos de medicamen- 
tos e dispensação de insumos em pediatria. Salvador, 2015, Hospital Santa Izabel (trabalho interno, artigo em construção).

SANTOS, G.M.M. Materiais e Medicamentos: vetores de custo no setor de saúde suplementar. Série IESS, 0046, 2013, disponível em: http://www.iess. org.br/TDIESS00462013MatMed.pdf, acesso em 17/11/2016, às 21:45.

Saúde Web: Saúde Suplementar. Operadoras: aumento dos gastos exige novo modelo, 20 de mar 2014; disponível em: http://saudebusiness.com/noticias/ operadoras-aumento-dos-gastos-exige-novo-modelo/, acesso em 29/10/2016.

ZUCCHI, P. et al. Gastos em saúde: os fatores que agem na demanda e na oferta dos serviços de saúde. 2000. Disponível em: http://www.scielo.br/pdf/sausoc/ v9n1-2/10.pdf , acesso em 25/11/16 às 19:15.

1- Serviço de Ortopedia do Hospital Santa Izabel

Endereço para correspondência:

barbara.edu@gmail.com 